\title{
Research on Intelligent Control System of Scaffold Based On PLC
}

\author{
Zhen Chen ${ }^{1,2}$, Jue Lu ${ }^{1+}$ Jian Song ${ }^{1,2}$ and Wei-ye Tian ${ }^{1}$ \\ ${ }^{1}$ School of Information Engineering, Wuhan University of Technology, Wuhan, China \\ ${ }^{2}$ Key Laboratory of Fiber Optic Sensing Technology and Information Processing (Wuhan University of \\ Technology), Ministry of Education, Wuhan, China
}

\begin{abstract}
The attached self-lifting scaffold is a new emerging scaffold technology for high-rise buildings. Generally speaking, because of the partial load, the deviation of hoist itself and environmental factors, scaffold in the process of lifting will appear the asynchrony problem. Aiming at this problem, the paper puts forward a method of using fuzzy control thinking which can adjust multiple hoists speed at the same time, so it can make the scaffolding stay at the synchronous state. The system applies the sensor to measure the tension value of each lifting point. After it is processed by the fuzzy control, it can use its reasoning ability to get the frequency value which the hoist needs to adjust. Using the way of USS communication to send the frequency value acquired by the reasoning to each inverter, so that it can change the lifting speed of each hoist. At last, the load of each position tends to be the same. It has been used by simulation experiment and the engineering application, which can verify that the intelligent control system has better effect on synchronous control in the scaffold lifting process.
\end{abstract}

Keywords: PLC, fuzzy control, scaffold, inverter, USS communication

\section{Introduction}

The attached self-lifting scaffold, used for the construction industry, is an intelligent tool. Its control system can make the scaffold to rise and fall according to the building structure along with the need to work. Because the scaffold is a rigid structure, the difference in lifting speed of the motor will directly lead to the scaffolding hoisting status inconsistency, which causes uneven distribution of load and the changes of tension [1]. The value of tensile as the basis for judging the scaffold balance not only provide a direct response to the actual state of the scaffold but also play the role of protection and early warning. Traditional control systems typically need to stop to check the state of each position, and to re-adjust the hoists to equilibrium after finding the cause. It is difficult to establish accurate mathematical model in scaffold control system, traditional control theory cannot be made good effect. In response to this problem, this paper combined with the theory of fuzzy control, frequency control technology and sensor technology, designed an automatic control system on the basis of PLC control system [2]. This system in the scaffold lifting process relative to the traditional control system has a better synchronization effect.

\section{Fuzzy control system}

\subsection{Basic structure}

Lifting scaffold control system is mainly composed of the host computer, PLC (Programmable Logic Controller), inverter, hoist, data acquisition module and other components. We have installed tension sensors at each lifting position of the scaffold, which measure the load value of the lifting point in real time and display it in the touch screen used to observe and monitor the system state. The sensor will send the measured data to the PLC for comprehensive judgment. If error obtained exceeds the allowable range, then

\footnotetext{
+ Corresponding author. Tel.: +8618971454310.

E-mail address: xiaozhen26828@126.com.
} 
immediately stop the hoists running and send an alarm signal. When the error is within the allowable range, the data will be processed by fuzzy control algorithm, and we can get the output control of lifting point [3]. Finally, system use the inverter to change the speed of the hoists to correct the lifting attitude. The basic structure is as follows.

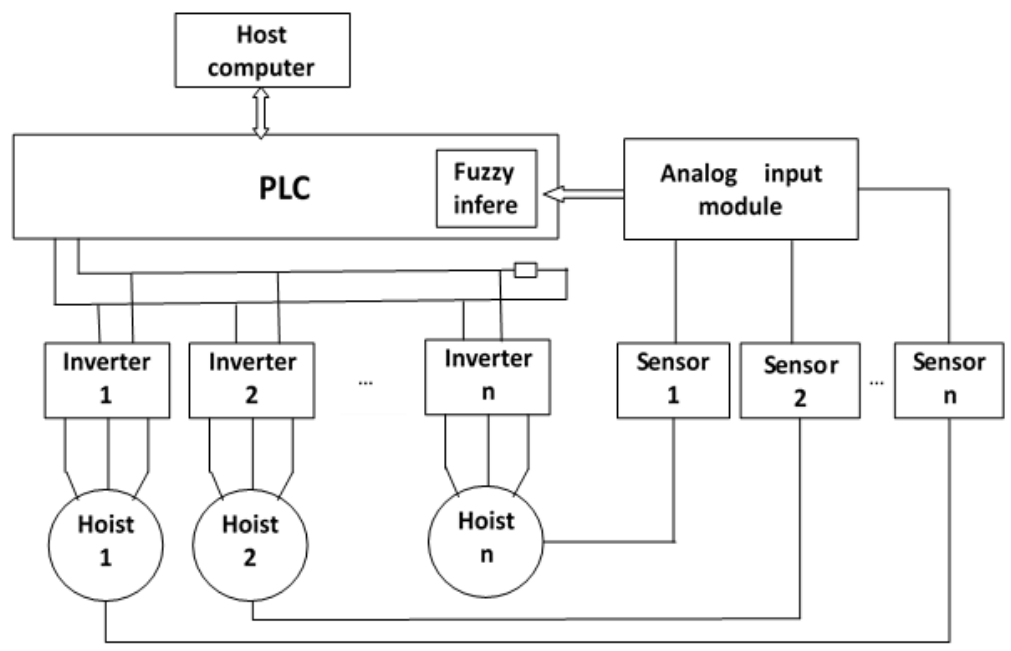

Fig. 1: System structure diagram.

\subsection{Fuzzy Control Design}

The core of control system is the fuzzy controller. It will calculate the appropriate amount of control depending on the error and the variety rate of error, so that the control achieve the desired effect. The process of fuzzy control mainly includes fuzzification, fuzzy inference, rule base and defuzzification. The difference between the actual tension value and the set value is the error, and difference between this error and the previous error is variety rate of error. The control system selects the error(E) and the variety rate of error(EC) as the input variable, and the frequency $(\Delta \mathrm{U})$ is used as the output variable. We define Negative Big (NB), Negative Middle (NM), Negative Small (NS), Zero (O), Positive Small (PS), Positive Middle (PM), and Positive Big (PB) as linguistic variables of fuzzy subset. The control rule is the most important part of the fuzzy controller, and it's correct or not directly affects the performance of the controller. We define fuzzy control rule table based on actual work and expert experience. The table is as follows.

Table 1: Fuzzy Control Rules

\begin{tabular}{|c|c|c|c|c|c|c|c|}
\hline$\Delta$ & $\mathrm{NB}$ & NM & NS & $\mathrm{O}$ & PS & $\mathrm{PM}$ & PB \\
\hline NB & PB & PB & PM & PS & PS & $\mathrm{O}$ & $\mathrm{O}$ \\
\hline $\mathrm{NM}$ & PB & PB & PM & PS & PS & $\mathrm{O}$ & NB \\
\hline NS & PB & PB & $\mathrm{PM}$ & PS & $\mathrm{O}$ & PS & $\mathrm{NB}$ \\
\hline $\mathrm{O}$ & PB & $\mathrm{PM}$ & PS & $\mathrm{O}$ & NS & $\mathrm{NM}$ & $\mathrm{NB}$ \\
\hline PS & PM & $\mathrm{PM}$ & $\mathrm{O}$ & NS & $\mathrm{NM}$ & PB & PB \\
\hline PM & PS & $\mathrm{O}$ & NS & $\mathrm{NM}$ & $\mathrm{NM}$ & NB & $\mathrm{NB}$ \\
\hline PB & $\mathrm{O}$ & $\mathrm{O}$ & NM & NB & NB & NB & NB \\
\hline
\end{tabular}

The precise control input is converted into fuzzy quantity by the fuzzy process. The fuzzy interrogation table is extracted from fuzzy-rule by using off-line calculating method. The elements of the table will be stored in the PLC memory area, according to the order of top to bottom and left to right. Finally, fuzzy control output quantity can be queried through online method. We use the method of address addressing to 
look up the table. The address of the fuzzy control quantity according to E and EC values calculated directly obtained. Fuzzy control flow chart is as follows.

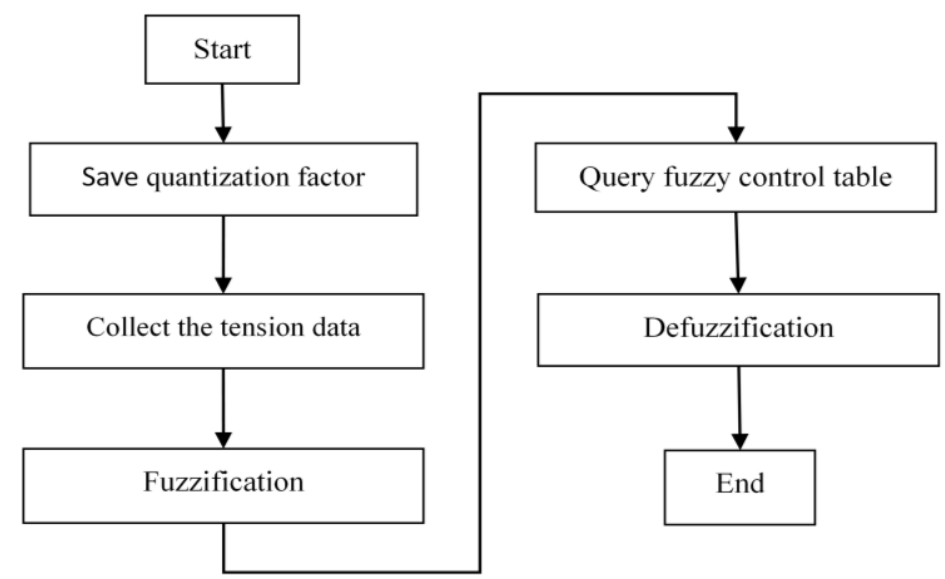

Fig. 2: Fuzzy control flow chart.

\subsection{Communication Network Design}

The method of using PLC to control the inverter is achieved by RS-485 communication. This method allows the connection between the inverter and the PLC is greatly reduced, reducing interference and signal transmission errors in the system. We choose USS (Universal Serial Interface Protocol) communication protocol to transfer data [4]. This protocol enables one primary station to communication with many passive stations at once, and the number of passive stations can be tall up to 31.If there are various passive stations, in order to facilitate normal communication, we have to set the address for each passive station. The primary station can find it by the passive station address. During the process of communication, the passive station cannot send the information to the primary station before receiving the request from the primary station [5]. Only if it has received information from the primary station, it can reply to the primary station according to the received information. The main program used for communication between PLC and inverter are as follows.
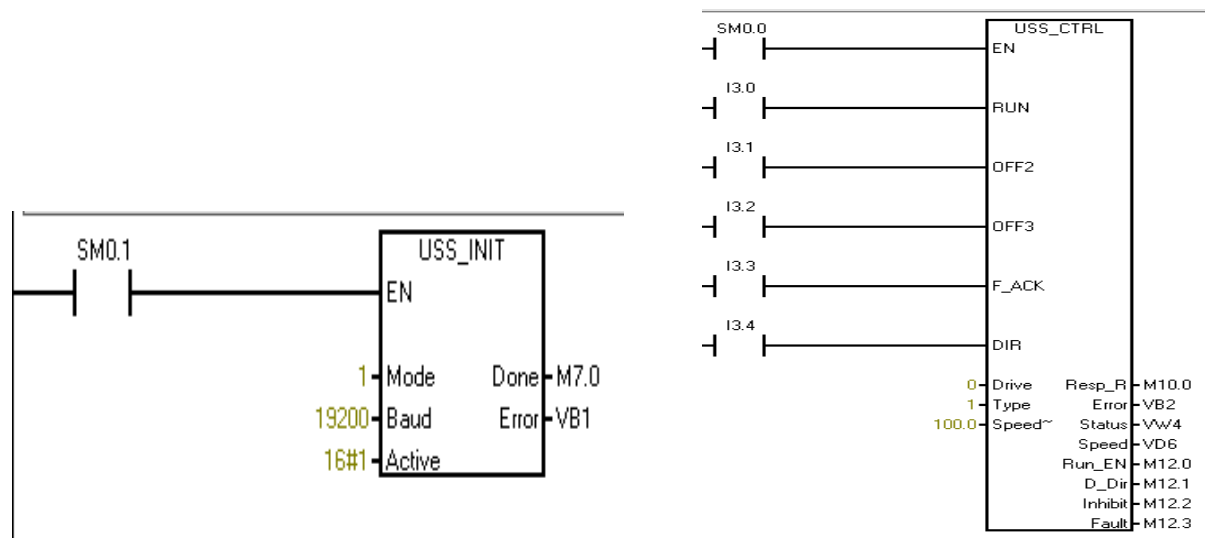

Fig. 3: Initializing instruction and control inverter instruction.

Where, initializing instruction is used for setting communication parameters. The "Active" is the address of the inverters that need communication. "Active" is a 32-bit integer, if one of the number is 1,then this address on behalf of the inverter will be activated. For example, if the value of "Active" is $16 \# 00000 \mathrm{FFF}$, then the inverter with the address of 0 to 11 are activated [6].

Control inverter instruction is used for control inverter. Its main function is making the inverter in different running state. 

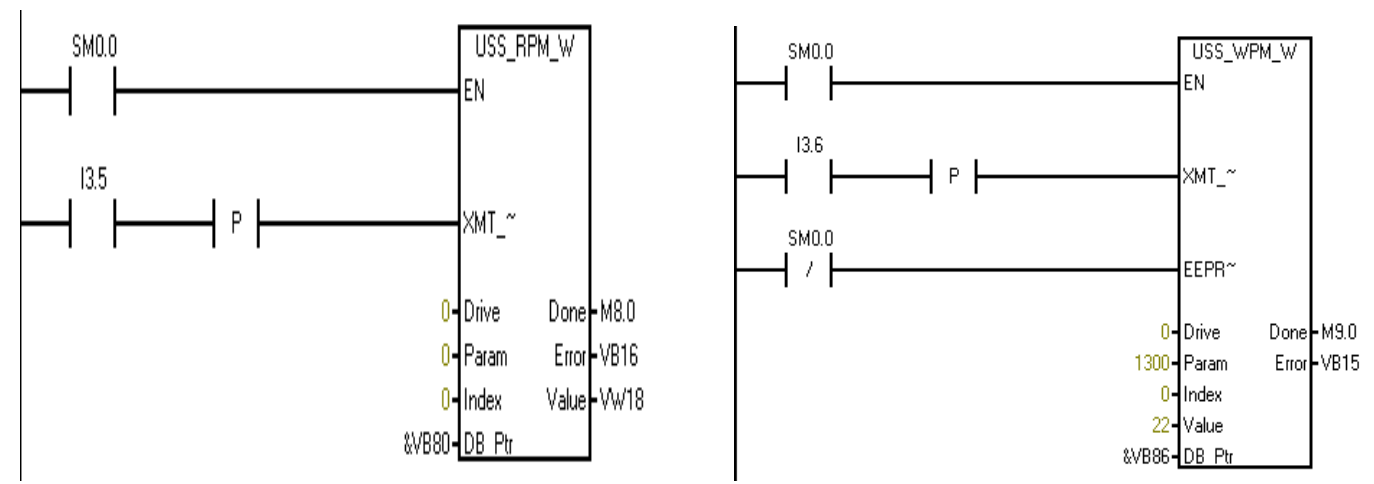

Fig. 4: Read inverter instruction and Write inverter instruction.

Where, read inverter instruction is used for reading parameters from inverter. The "Value" is the data that PLC will be read from inverter. Write inverter instruction is used for writing parameters to inverter. The "Value" is the data that will be sent to the inverter.

\subsection{Simulation}

In order to test the performance of the system in control, we have done a simulation experiment on both our fuzzy control system and traditional PID control system through MATLAB software. We add an interfering signal at $300 \mathrm{~ms}$ to test the anti-interference ability of the system. The simulation results are as follows.
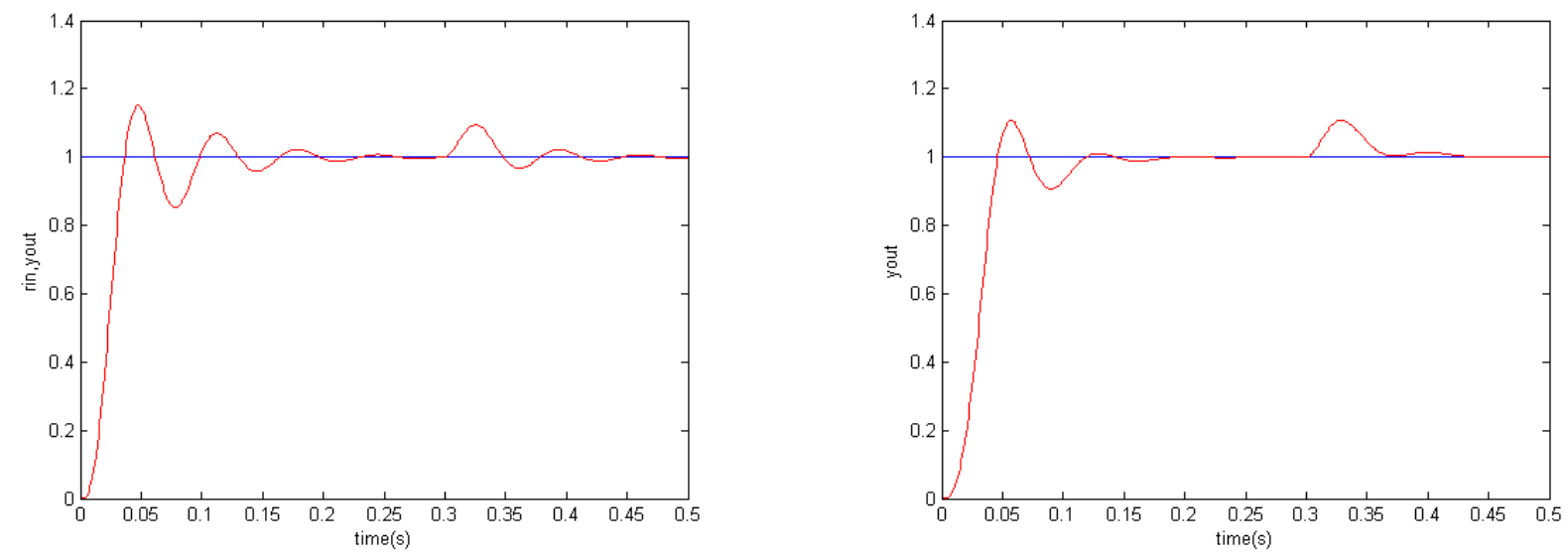

Fig. 5: Simulation results.

Left figure is the result of traditional PID control system and right figure is the result of fuzzy control system. As you can see from the results, the fuzzy control system has the advantages of smaller overshoot, fast response and stronger robustness. Through the simulation experiment, we know that using fuzzy control to achieve the control effect is better than using classic PID control mode.

\section{Conclusion}

With the rapid development of intelligent control technology, PLC has been widely used in industry. In this paper, the system we design can solve the problem of scaffold lifting synchronization. The experimental results show that our system control effect is better than the traditional method. The fuzzy control theory improves the intelligent level of the system, and USS communication makes one PLC to control multiple inverters. It has been verified in a company in Wuhan.

\section{Acknowledgements}

First of all, I would like to express my gratitude to all those who helped me during the writing of this paper. Their help enabled me to finish the work successfully.

\section{References}


[1] K.M. Halperin, M. Mccann. An evaluation of scaffold safety at construction sites. Journal of Safety Research, 2004, 35(2), 141-50.

[2] A.M. Harb, I. A. Smadi. An Approach To Fuzzy Control For A Class Of Nonlinear Systems: Stability And Design Issues. International Journal of Modelling \& Simulation.2005, 25(2): 106-111.

[3] K.M. Passino, S. Yurkovich. Fuzzy Control. Tsinghua University Press, 2001.

[4] M.G. Ioannides. Design and implementation of PLC-based monitoring control system for induction motor. IEEE Transactions on Energy Conversion.2004, 19(3):469-476.

[5] X. Song, S. Tan, and J. Ding. A Monitoring System for PLC Controlled Manufacturing System Based on Fieldbus. Knowledge Enterprise: Intelligent Strategies in Product Design, Manufacturing, and Management. Springer US. 2006, pp.576-581.

[6] X.U. Jia-Ming. Design plc standard communication program which communicates frequency converter based on uss protocol. Microcomputer Information. 2009, 25(3), 164-166. 